\title{
The Significance of Insulin Resistance in Nondiabetic Breast Cancer Patients
}

\author{
Berrin Papila Kundaktepe ${ }^{\mathrm{a}}$, Sinem Durmus ${ }^{\mathrm{b}}$, Mahir Cengiz ${ }^{\mathrm{c}}$, Fatih Orkun Kundaktepe ${ }^{\mathrm{d}}$, \\ Volkan Sozere $^{\mathrm{e}}$, Cigdem Papila ${ }^{\mathrm{f}}$, Remise Gelisgen ${ }^{\mathrm{b}}$, Hafize Uzun ${ }^{\mathrm{b}, \mathrm{g}}$
}

\begin{abstract}
Background: The relationship between insulin resistance (IR) and prognostic factors in breast cancer (BC) in premenopausal (pre-M) and postmenopausal (post-M) women is still controversial. We evaluated the potential association between hemoglobin A1c (HbAlc), fasting blood glucose (FBG), fasting insulin levels (FILs), the homeostasis model assessment index (HOMA), and the prognostic factors of $\mathrm{BC}$ in nondiabetic patients with pre-M and post-M breast tumors.
\end{abstract}

Methods: We compared 80 nondiabetic patients with pre-M and post-M breast tumors to 60 women with normal mammography as a control.

Results: Age, body mass index (BMI), FBG, and HbA1c did not differ between the groups. FIL $(\mathrm{P}<0.001)$ and HOMA-IR $(\mathrm{P}<0.001)$ of the $\mathrm{BC}$ group were significantly higher than in the control group. FIL $(\mathrm{P}<0.001)$ and HOMA-IR $(\mathrm{P}<0.001)$ of the BC group were significantly higher than in the control group, for both pre- and post$M$ patients. FIL and HOMA-IR values were found to be significantly higher in the patients with stage IV BC than in other stages of BC. FIL and HOMA-IR are highly specific and sensitive parameters in their ability to diagnose BC.

Conclusions: FIL and HOMA-IR are associated with $\mathrm{BC}$ risk in nondiabetic patients with pre-M and post-M breast tumors. When BC

Manuscript submitted February 11, 2021, accepted March 12, 2021

Published online April 25, 2021

aDepartment of Surgery, Cerrahpasa Medicine Faculty, Istanbul UniversityCerrahpasa, Istanbul, Turkey

bDepartment of Medical Biochemistry, Cerrahpasa Faculty of Medicine, Istanbul University-Cerrahpasa, Istanbul, Turkey

${ }^{\mathrm{c}}$ Department of Internal Medicine, Biruni University School of Medicine, Istanbul, Turkey

${ }^{\mathrm{d} D e p a r t m e n t}$ of Internal Medicine, Gaziosmanpasa Taksim Research and Training Hospital, Istanbul, Turkey

eDepartment of Biochemistry, Yildiz Technical University, Istanbul, Turkey

fDepartment of Internal Medicine, Division of Oncology, Cerrahpasa Faculty of Medicine, Istanbul University-Cerrahpasa, Istanbul, Turkey

'Corresponding Author: Hafize Uzun, Department of Medical Biochemistry, Cerrahpasa Faculty of Medicine, Istanbul University-Cerrahpasa, Cerrahpasa 34303, Istanbul, Turkey. Email: huzun59@hotmail.com

doi: https://doi.org/10.14740/jem729 risk was evaluated according to the stage of menopause, no difference was observed; only the disease stage was significant. FIL and IR may function as potential biomarkers and therapeutic targets for human cancers.

Keywords: Breast cancer; HbA1c; Fasting blood glucose; Fasting insulin; Insulin resistance

\section{Introduction}

Breast cancer (BC) is the most commonly diagnosed cancer among women. It is also in the second place among female cancer patients in cancer-related deaths; and mortality rates are expected to increase further in the next decade [1].

Insulin resistance (IR) is a pathologic clinical condition progressing with hyperinsulinemia. Hyperinsulinemia doubles the risk of $\mathrm{BC}$ in postmenopausal (post-M) women $[2,3]$. IR takes a role in the pathogenesis of type 2 diabetes mellitus (T2DM), obesity, and cancer. Women with IR syndrome characteristics such as obesity, high insulin levels, diabetes, and physical inactivity are at increased risk of $\mathrm{BC}[4,5]$. It should be noted that insulin is not a parameter indicating the future incidence of BC. However, it is thought that there may be a weak relationship between $\mathrm{BC}$ and T2DM [5].

The mechanical relationship between overweight and negative $\mathrm{BC}$ is linked to IR and fasting insulin level (FIL)-related pathways. Obesity poses different BC risks in premenopausal (pre-M) and post-M women, depending on estrogen receptor (ER) status [6]. Genetic variants related to IR can affect the risk of $\mathrm{BC}[7]$.

Our goal in this research was to clarify the potential relationship between hemoglobin A1c (HbAlc), fasting blood glucose (FBG), FILs, and IR, and the prognostic factors of BC in nondiabetic patients with pre-M and post-M breast tumors.

\section{Materials and Methods}

This was a retrospective case-control study conducted at the Department of General Surgery and the Department of Internal Medicine, approved by the Ethics Committee of Istanbul University-Cerrahpasa, Cerrahpasa Medical Faculty. Eighty con- 
secutive nondiabetic patients with pre-M and post-M breast tumors and 60 consecutive age-matched, pre-M and post-M healthy control subjects were enrolled in this research.

All subjects were selected from people of Turkish origin. Pregnant women, patients with renal, hepatic, rheumatic or endocrine diseases were not included in the study. In addition, smokers, those with a history of chronic alcohol consumption, and subjects taking oral antidiabetics such as metformin, dipeptidyl peptidase-4 (DPP-4) inhibitors, glucagon-like peptide-1 (GLP-1) analogues, hepatotoxic drugs (antituberculosis), and subjects taking antiepileptic or oral contraceptive pills were also excluded from the study. For cancer staging, the tumor-node-metastasis (TNM) classification established by the American Joint Cancer Committee (AJCC) was used [8].

Healthy subjects without any endocrine, vascular, cardiac or inflammatory diseases were selected as the control group. None of the subjects has a family history of diabetes. None of the subjects had diabetes or glucose intolerance as confirmed by the oral glucose tolerance test (OGTT).

Anthropometric characteristics (weight, height) of the individuals were recorded. Body mass index (BMI) of the subjects was calculated using the formula: BMI $\left(\mathrm{kg} / \mathrm{m}^{2}\right)=$ weight $(\mathrm{kg}) /(\text { height }(\mathrm{m}))^{2}[9]$.

Demographic (age, gender) and clinical characteristics of individuals (comorbid conditions, primary tumor size, histological subtype, axillary lymph node involvement, TNM stage, ER or progesterone receptor (PR) status, human epidermal growth factor receptor-2 (HER-2) status and histological grade) were obtained from the medical records of individuals.

The dilution was 1/100 for ER (Novocastra, Leica Biosystems, Nussloch, Germany; clone 6F11), 1/75 for PR (Novocastra, Leica Biosystems; clone 312), and 1/80 for HER-2 (Novocastra, Leica Biosystems; clone CB11).

Blood samples were taken from the participants before surgery. After an overnight fasting, they were taken into tubes containing anticoagulant (ethylenediamine tetraacetic acid (EDTA)) and tubes without anticoagulant. Immediately after venous blood collection, blood samples were centrifuged at $4{ }^{\circ} \mathrm{C}$ for $10 \mathrm{~min}(3,000 \mathrm{~g})$ and plasma and serum were obtained. Glucose levels were determined by enzymatic methods (Abbott Diagnostics, Abbott Park, IL, USA). FIL were measured by an electrochemiluminescence immunoassay (ECLIA) method on a Roche-Hitachi E170. HbA1c levels were assessed by high performance liquid chromatography (HPLC) (Bio-Rad Variant 2 Turbo). All biochemical parameters were obtained from the patients' medical records.

FBG and FIL were measured in subjects, and these values were used to determine the degree of IR in conjunction with homeostasis model assessment (HOMA). HOMA-IR was calculated using the following formula: HOMA-IR $=(\mathrm{FBG}(\mathrm{mg} /$ $\mathrm{dL}) \times \operatorname{FIL}(\mu \mathrm{U} / \mathrm{mL})) / 405[10]$.

\section{Statistical analysis}

Distribution was tested using the single sample KolmogorovSmirnov test. Categorical variables were presented as the frequency and percentage and analyzed by using Chi-square test. Numerical variables were expressed as mean \pm standard deviation and analyzed by using two-sided Student's $t$ - test. Receiver operating characteristic (ROC) curve analysis was performed. Statistical significance limit was accepted as 0.05. Analyzes were performed using SPSS 20.0 software for Windows (SPSS, Chicago, IL, USA).

\section{Results}

The demographic data and laboratory findings of the studied groups are shown in Table 1. Age, BMI, FBG, and HbA1c did not differ among the groups. FIL $(\mathrm{P}<0.001)$ and HOMA-IR ( $\mathrm{P}$ $<0.001)$ levels of the BC group were significantly higher than controls. In addition, Table 1 displays also the frequency of $\mathrm{BC}$ patients belonging to TNM, ER status, PR status, HER-2/neu status, and molecular subtype's classification.

In Table 2, each of the BC and control groups is divided into pre-M and post-M subgroups. When the demographic and biochemical data for these subgroups were compared, age difference was found between pre-M and pre-M control individuals $(\mathrm{P}<0.001)$. The age of post-M BC patients was significantly higher than that of the pre-M BC group $(\mathrm{P}<0.001)$. FIL and HOMA-IR values of pre-M BC patients were statistically significantly higher than those of pre-M control individuals (each $\mathrm{P}<0.001$ ); likewise, FIL and HOMA-IR values of post-M BC patients were found to be statistically significantly higher than those of post-M control individuals (each $\mathrm{P}<0.001$ ).

The ROC analysis results of FIL, FBG, HbAlc, and HOMA-IR levels for BC versus control are compared in Table 3. FIL and HOMA-IR had AUC values of 0.987 (sensitivity $98.8 \%$, specificity $82.7 \%$, and cutoff 8.5 ) and 0.998 (sensitivity $97.5 \%$, specificity $96.7 \%$, and cutoff 2.4 ), respectively, which demonstrates their sufficiency in distinguishing $\mathrm{BC}$ from control.

FIL $(\mathrm{P}<0.001)$ and HOMA-IR $(\mathrm{P}<0.001)$ levels of $\mathrm{BC}$ group according to TNM stage were significantly higher in the stage IV group (Table 4). A re-analysis of the biochemical parameters of ER, PR, and HER-2 in the BC subgroups revealed no change in the statistical significance of FIL and HOMA-IR (Table 5).

\section{Discussion}

IR is often overlooked because it is not classified as a disease. This study provides evidence that FIL and IR estimated using the HOMA were increased in nondiabetic patients with pre-M and post-M breast tumors. When FIL and IR were evaluated according to the stage of menopause, no difference was found; it was the disease stage that was significant. FIL and HOMAIR had a sensitivity of $98.8 \%$ and specificity of $82.7 \%$, and a sensitivity of $97.5 \%$ and specificity of $96.7 \%$, respectively, which demonstrates their sufficiency in distinguishing $\mathrm{BC}$ from healthy subjects. IR plays an important role in FIL homeostasis related to cancer.

It is known that IR, which is considered as the primary factor in metabolic syndrome mechanisms, also increases the risk of cardiovascular disease (CVD). IR is a key player in the pathogenesis of metabolic diseases and can be observed in 
Table 1. Demographic and Biochemical Parameters of the Groups

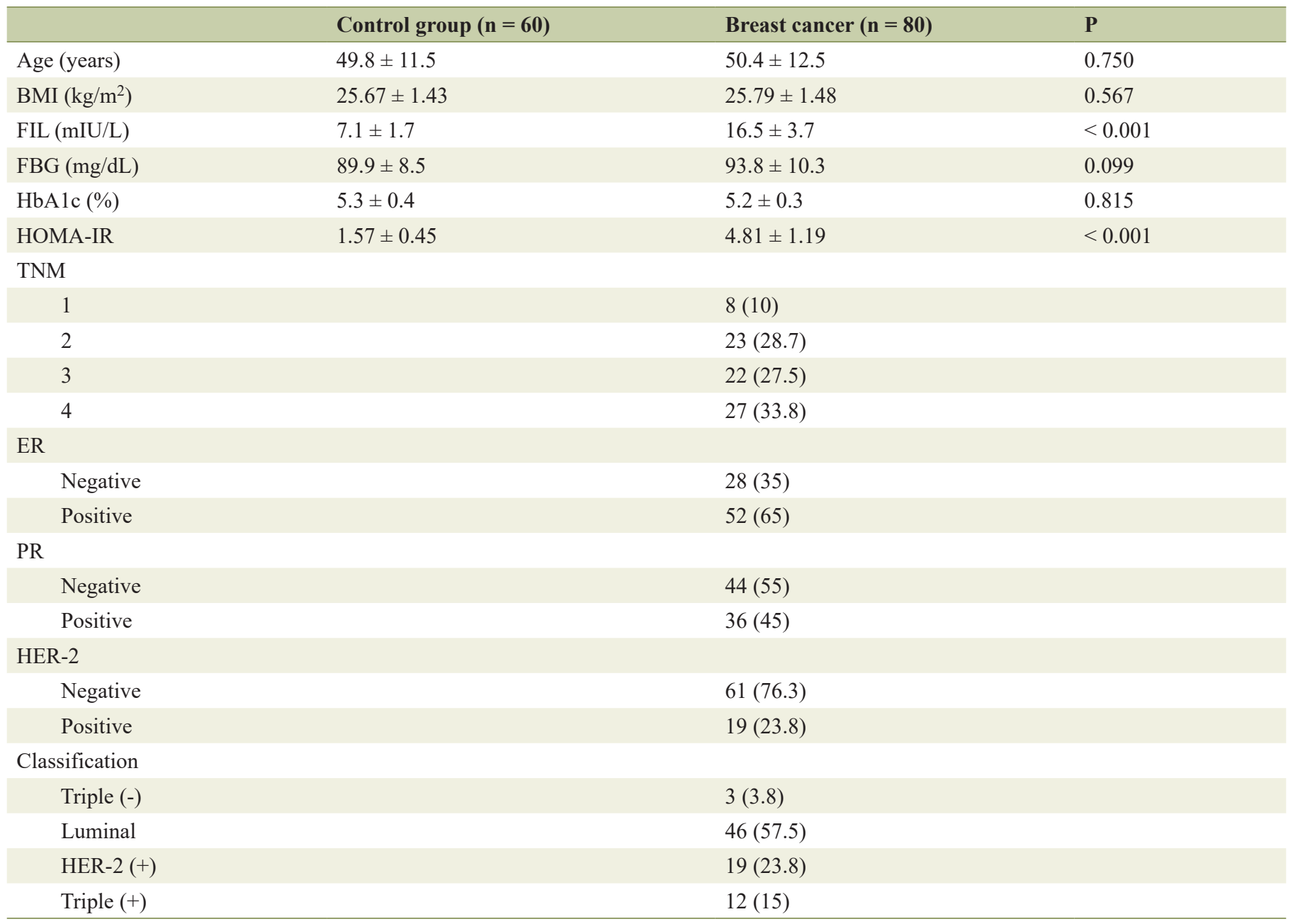

BMI: body mass index; FBG: fasting blood glucose; FIL: fasting insulin level; HbA1c: hemoglobin A1c; HOMA-IR: homeostasis model assessmentinsulin resistance; TNM: tumor-node-metastasis; ER: estrogen receptor; PR: progesterone receptor; HER-2/neu: human epidermal growth factor receptor-2.

several clinical conditions such as $\mathrm{BC}[11,12]$. The causative association between $\mathrm{BC}$ and IR is also controversial [13]. Ferroni et al [14] reported that it showed increased pretreatment FBG and FIL in nondiabetic women with $\mathrm{BC}$; although the higher pretreatment levels were not pathological, they resulted in higher HOMA indexes. In a meta-analysis, Hernandez et al [15] reported that they could not find any change in IR markers in BC patients. Since both factors are directly related to IR and

Table 2. Demographic and Biochemical Parameters of the Groups for Menopause

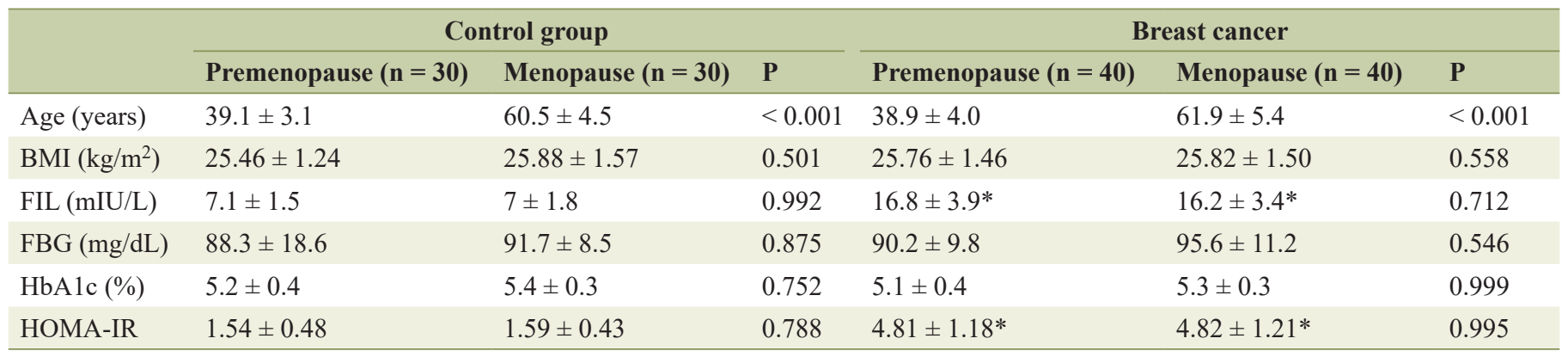

Comparison with control group, ${ }^{*} \mathrm{P}<0.001$. BMI: body mass index; FBG: fasting blood glucose; FIL: fasting insulin level; HbA1c: hemoglobin A1c; HOMA-IR: homeostasis model assessment-insulin resistance. 
Table 3. Sensitivity, Specificity, AUC, Cutoff, and Asymptotic Significance of Parameters in Study Groups (Control vs. Breast Cancer)

\begin{tabular}{lllllll} 
& Sensitivity (\%) & Specificity (\%) & AUC & Cutoff & P & $<0.001$ \\
FIL (mIU/L) & 98.8 & 82.7 & 0.987 & 8.5 & $<.4$ & 0.001 \\
HOMA-IR & 97.5 & 96.7 & 0.998 & 2.4 & \\
\hline
\end{tabular}

FIL: fasting insulin level; HOMA-IR: homeostasis model assessment-insulin resistance; AUC: area under the curve.

Table 4. Biochemical Parameters of the Breast Cancer Group for TNM Stage

\begin{tabular}{lllllll} 
& \multicolumn{4}{c}{ TNM } & & \\
\cline { 2 - 6 } & $\mathbf{1}$ & $\mathbf{2}$ & $\mathbf{3}$ & $\mathbf{4}$ & \\
\hline FIL (mIU/L) & $10.3 \pm 1.4$ & $14.9 \pm 3.4$ & $17.4 \pm 2.2$ & $19 \pm 2.2$ & $<0.001$ \\
HOMA-IR & $2.7 \pm 0.4$ & $4.1 \pm 0.7$ & $5.1 \pm 0.7$ & $5.9 \pm 0.6$ & $<0.001$ \\
\hline
\end{tabular}

FIL: fasting insulin level; HOMA-IR: homeostasis model assessment-insulin resistance; TNM: tumor-node-metastasis.

$\mathrm{BC}$, these studies may be biased by incorrect patient stratification in terms of BMI and menopausal status [15].

In our study, BMI, FBG, and HbAlc concentrations did not differ between patients and control subjects, nor did FIL, FBG, HbA1c, or HOMA-IR differ between the pre-M and postM BC groups. No relation was found between FIL and IR and prognostic factors, such as ER, PR, and HER-2, in nondiabetic patients with pre-M and post-M breast tumors. Brown et al [16] showed that FBG levels and HOMA-IR scores were higher in post-M women than in pre-M women. Luque et al [13] found that $\mathrm{BC}$ presence is associated with IR in overweight or obese pre-M women but not in pre-M or post-M women of normal weight. Tumor and IR markers are associated with impaired glucose/insulin metabolism in overweight or obese pre-M BC patients. This indicates a bidirectional relationship between irregular or unbalanced glucose/insulin metabolism data and BC. Yadav et al [17] noted significant differences in the HbA1c level, FIL, and C-peptide concentration of pre-M women between cases and controls. Insignificant results were found for FBG and BMI. Similarly, significant differences in FBG, FIL, C-peptide concentration, and HbA1c level were observed in post-M patients and controls. Similarly to our study, Manjer et al [18] found no significant differences in BC risk for perimenopausal (peri-M) and post-M women from different BMI quartiles and FBG levels. The association between
$\mathrm{BC}$ presence and IR was independently influenced by BMI, ER, PR, and HER-2 in nondiabetic peri-M and post-M women. The role of FIL, FBG, and IR as markers for the diagnosis of $\mathrm{BC}$ in peri-M and post-M women must be widely evaluated.

Insulin has been shown to stimulate cell proliferation in normal breast tissue and human BC cell lines [19, 20], and to enhance breast tumor growth in animal models [21, 22]. Insulin growth factors (IGF) and IGF binding proteins (IGFBP) are valuable factors for work as well as FIL. It is emphasized that IGF1 and IGF1/IGFBP3 ratios may be parameters associated with mammographic density and the risk of BC development $[23,24]$. IGF1, released by adipocytes and regulated by glucose and fatty acids, has been reported to play a role in the control of cancer cell growth in obese individuals [25]. IGF1 concentrations and sex steroids and plasma sex hormone binding globulin (SHBG) concentrations are thought to be factors that may explain this association between cancer and obesity [26]. Clinical studies have also shown an association between hyperinsulinemia, IR and SHBG [27-29]. Insulin and IGF1 inhibit the hepatic synthesis of SHBG. By reducing SHBG levels, insulin exerts a positive effect on estrogen bioavailability, thereby increasing BC risk [30]. In our study, FIL and HOMAIR of the $\mathrm{BC}$ group were significantly higher than those of the control group. FIL and HOMA-IR of the BC group according to TNM stage were significantly higher in the stage IV group

Table 5. Biochemical Parameters of the Breast Cancer Group for ER, PR and HER-2

\begin{tabular}{llll} 
& PR $(-),(\mathbf{n}=\mathbf{4 4})$ & PR $(+),(\mathbf{n}=\mathbf{3 6})$ & P \\
\hline FIL (mIU/L) & $16.3 \pm 3.9$ & $16.7 \pm 3.4$ & 0.645 \\
HOMA-IR & $4.8 \pm 1.3$ & $4.9 \pm 1.1$ & 0.616 \\
& ER $(-),(\mathrm{n}=28)$ & ER $(+),(\mathrm{n}=52)$ & P \\
FIL (mIU/L) & $16.7 \pm 3.8$ & $16.4 \pm 3.6$ & 0.767 \\
HOMA-IR & $4.9 \pm 1.2$ & $4.8 \pm 1.2$ & 0.556 \\
& HER-2 $(-),(\mathrm{n}=61)$ & HER-2 $(+),(\mathrm{n}=19)$ & P \\
FIL (mIU/L) & $16.2 \pm 3.6$ & $17.6 \pm 3.8$ & 0.127 \\
HOMA-IR & $4.7 \pm 1.2$ & $5.2 \pm 1.1$ & 0.115 \\
\hline
\end{tabular}

FIL: fasting insulin level; HOMA-IR: homeostasis model assessment-insulin resistance; ER: estrogen receptor; PR: progesterone receptor; HER2/neu: human epidermal growth factor receptor-2. 
than in the other stages. FIL and HOMA-IR had a sensitivity of $98.8 \%$ and specificity of $82.7 \%$, and a sensitivity of $97.5 \%$ and specificity of $96.7 \%$, respectively, which demonstrates their sufficiency in distinguishing BC from control. The results of our study were consistent with some other studies [2, 3, 3133]. However, some results in the literature are contradictory. No consistent association was found between FIL and BC [5, 34-35]. Del Giudice et al [31] found that FIL and IGFBP-3 levels were elevated in women with pre-M BC, regardless of diet and other known risk factors for $\mathrm{BC}$. This finding may indicate the presence of an underlying IR syndrome independent of obesity. Gunter et al [3] stated that hyperinsulinemia is an independent risk factor for BC and may be the basis for explaining the relationship between obesity and BC. Jernstrom et al [34] reported that proinsulin, FIL and C-peptide levels were positively correlated with current weight and weight gain, but these hormones and IGF1 levels did not differ between women with and without BC. Past estrogen replacement therapy (ERT) was more common among women with BC and the duration of use was longer. It has been emphasized that the risk of $\mathrm{BC}$ increases significantly in women who gain weight or use hormone replacement therapy (HRT), but this increased risk is not related to circulating levels of IGF1, FIL, proinsulin or Cpeptide. Kaaks et al [35] reported that there was no clear relationship between BC risk and FIL. Mink et al [5] reported that FIL do not predict future incidence of $\mathrm{BC}$, but may be weakly associated with T2DM, perhaps modulated through increased adiposity. Given these conflicting results, the effects of insulin on $\mathrm{BC}$ need to be further investigated [36].

In one study conducted in 2020, Pan et al [37] found that increased IR in post-M women is associated with higher BC incidence and higher all-cause mortality after BC. Another study found that IR may have a negative effect on pathological complete response following neoadjuvant therapy particularly with hormone-positive and HER-2-negative cases of nondiabetic BC [38]. Despite all speculation, Yee et al [39] recommended that oncologists care about insulin because of "Given the important role insulin signaling plays in driving signaling pathways that promote aggressive cancer biology, more attention should be paid by cancer physicians to screening and treating IR."

The outcomes of this research suggest that insulin may be one of the contributing factors (excluding obesity) to the development of BC. A high FIL is a potential risk factor for BC development in nondiabetic women. IR is an interrupted state in the biological response to insulin in nondiabetic patients with pre-M and post-M breast tumors. Because insulin may affect $\mathrm{BC}$ risk and prognosis, it is important to control IR. Considering that the insulin signal activates the signaling pathways in cancer biology, the treatment of IR with medical and integrative approaches may be useful in cancer treatment. FIL and IR may function as potential biomarkers and therapeutic targets for human cancers, and their role should be further studied to improve our understanding of $\mathrm{BC}$ pathogenesis and progression.

\section{Acknowledgments}

None to declare.

\section{Financial Disclosure}

None to declare.

\section{Conflict of Interest}

None to declare.

\section{Informed Consent}

Written informed consent was obtained from each subject after they were informed about the study.

\section{Author Contributions}

BPK, CP, RG, and HU contributed to the conception and interpretation of the data. SD, MC, and VS performed the statistical analysis. BPK, SD, FOK, CP, RG, and $\mathrm{HU}$ were involved in writing, reviewing, and editing the manuscript. All the authors read and approved the final manuscript.

\section{Data Availability}

The data supporting the findings of this study are available from the corresponding author upon reasonable request.

\section{References}

1. Greaney ML, Sprunck-Harrild K, Ruddy KJ, Ligibel J, Barry WT, Baker E, Meyer M, et al. Study protocol for Young \& Strong: a cluster randomized design to increase attention to unique issues faced by young women with newly diagnosed breast cancer. BMC Public Health. 2015;15:37.

2. Kabat GC, Kim M, Caan BJ, Chlebowski RT, Gunter MJ, Ho GY, Rodriguez BL, et al. Repeated measures of serum glucose and insulin in relation to postmenopausal breast cancer. Int J Cancer. 2009;125(11):2704-2710.

3. Gunter MJ, Hoover DR, Yu H, Wassertheil-Smoller S, Rohan TE, Manson JE, Li J, et al. Insulin, insulin-like growth factor-I, and risk of breast cancer in postmenopausal women. J Natl Cancer Inst. 2009;101(1):48-60.

4. Goodwin PJ. Insulin resistance in breast cancer: relevance and clinical implications. Breast Cancer Research. 2011;13(2):1-9.

5. Mink PJ, Shahar E, Rosamond WD, Alberg AJ, Folsom AR. Serum insulin and glucose levels and breast cancer incidence: the atherosclerosis risk in communities study. Am J Epidemiol. 2002;156(4):349-352.

6. Picon-Ruiz M, Morata-Tarifa C, Valle-Goffin JJ, Friedman ER, Slingerland JM. Obesity and adverse breast cancer risk and outcome: Mechanistic insights and strategies 
for intervention. CA Cancer J Clin. 2017;67(5):378-397.

7. Jung SY, Papp JC, Sobel EM, Yu H, Zhang ZF. Breast Cancer Risk and Insulin Resistance: Post Genome-Wide Gene-Environment Interaction Study Using a Random Survival Forest. Cancer Res. 2019;79(10):2784-2794.

8. Giuliano AE, Connolly JL, Edge SB, Mittendorf EA, Rugo HS, Solin LJ, Weaver DL, et al. Breast Cancer-Major changes in the American Joint Committee on Cancer eighth edition cancer staging manual. CA Cancer J Clin. 2017;67(4):290-303.

9. World Health Organization. WHO mean body mass index (BMI). Retrieved February 5, 2019.

10. Matthews DR, Hosker JP, Rudenski AS, Naylor BA, Treacher DF, Turner RC. Homeostasis model assessment: insulin resistance and beta-cell function from fasting plasma glucose and insulin concentrations in man. Diabetologia. 1985;28(7):412-419.

11. Sieri S, Muti P, Claudia A, Berrino F, Pala V, Grioni S, Abagnato CA, et al. Prospective study on the role of glucose metabolism in breast cancer occurrence. Int J Cancer. 2012;130(4):921-929.

12. Goodwin PJ, Ennis M, Bahl M, Fantus IG, Pritchard KI, Trudeau ME, Koo J, et al. High insulin levels in newly diagnosed breast cancer patients reflect underlying insulin resistance and are associated with components of the insulin resistance syndrome. Breast Cancer Res Treat. 2009;114(3):517-525.

13. Luque RM, Lopez-Sanchez LM, Villa-Osaba A, Luque IM, Santos-Romero AL, Yubero-Serrano EM, Cara-Garcia $\mathrm{M}$, et al. Breast cancer is associated to impaired glucose/insulin homeostasis in premenopausal obese/overweight patients. Oncotarget. 2017;8(46):81462-81474.

14. Ferroni P, Riondino S, Laudisi A, Portarena I, Formica V, Alessandroni J, D'Alessandro R, et al. Pretreatment Insulin Levels as a Prognostic Factor for Breast Cancer Progression. Oncologist. 2016;21(9):1041-1049.

15. Hernandez AV, Guarnizo M, Miranda Y, Pasupuleti V, Deshpande A, Paico S, Lenti H, et al. Association between insulin resistance and breast carcinoma: a systematic review and meta-analysis. PLoS One. 2014;9(6):e99317.

16. Brown KA, Iyengar NM, Zhou XK, Gucalp A, Subbaramaiah K, Wang H, Giri DD, et al. Menopause Is a Determinant of Breast Aromatase Expression and Its Associations With BMI, Inflammation, and Systemic Markers. J Clin Endocrinol Metab. 2017;102(5):1692-1701.

17. Yadav NK, Poudel B, Thanpari C, Chandra Koner B. Assessment of biochemical profiles in premenopausal and postmenopausal women with breast cancer. Asian Pac J Cancer Prev. 2012;13(7):3385-3388.

18. Manjer J, Kaaks R, Riboli E, Berglund G. Risk of breast cancer in relation to anthropometry, blood pressure, blood lipids and glucose metabolism: a prospective study within the Malmo Preventive Project. Eur J Cancer Prev. 2001;10(1):33-42.

19. Ish-Shalom D, Christoffersen CT, Vorwerk P, SacerdotiSierra N, Shymko RM, Naor D, De Meyts P. Mitogenic properties of insulin and insulin analogues mediated by the insulin receptor. Diabetologia. 1997;40(Suppl 2):S2531.
20. Chappell J, Leitner JW, Solomon S, Golovchenko I, Goalstone ML, Draznin B. Effect of insulin on cell cycle progression in MCF-7 breast cancer cells. Direct and potentiating influence. J Biol Chem. 2001;276(41):3802338028.

21. Shafie SM, Grantham FH. Role of hormones in the growth and regression of human breast cancer cells (MCF-7) transplanted into athymic nude mice. J Natl Cancer Inst. 1981;67(1):51-56.

22. Shafie SM, Hilf R. Insulin receptor levels and magnitude of insulin-induced responses in 7,12-dimethylbenz(a)anthracene-induced mammary tumors in rats. Cancer Res. 1981;41(3):826-829.

23. Campagnoli C, Biglia N, Belforte P, Botta D, Pedrini E, Sismondi P. Post-menopausal breast cancer risk: oral estrogen treatment and abdominal obesity induce opposite changes in possibly important biological variables. Eur J Gynaecol Oncol. 1992;13(2):139-154.

24. Byrne C, Colditz GA, Willett WC, Speizer FE, Pollak M, Hankinson SE. Plasma insulin-like growth factor (IGF) I, IGF-binding protein 3, and mammographic density. Cancer Res. 2000;60(14):3744-3748.

25. D'Esposito V, Passaretti F, Hammarstedt A, Liguoro D, Terracciano D, Molea G, Canta L, et al. Adipocyte-released insulin-like growth factor-1 is regulated by glucose and fatty acids and controls breast cancer cell growth in vitro. Diabetologia. 2012;55(10):2811-2822.

26. Holly J. Insulin-like growth factor-1 and risk of breast cancer. Lancet. 1998;352(9137):1388.

27. Nestler JE. Sex hormone-binding globulin: a marker for hyperinsulinemia and/or insulin resistance? J Clin Endocrinol Metab. 1993;76(2):273-274.

28. Kalme T, Koistinen H, Loukovaara M, Koistinen R, Leinonen P. Comparative studies on the regulation of insulin-like growth factor-binding protein-1 (IGFBP-1) and sex hormone-binding globulin (SHBG) production by insulin and insulin-like growth factors in human hepatoma cells. J Steroid Biochem Mol Biol. 2003;86(2):197-200.

29. Lonning PE, Helle SI, Johannessen DC, Adlercreutz H, Lien EA, Tally M, Ekse D, et al. Relations between sex hormones, sex hormone binding globulin, insulin-like growth factor-I and insulin-like growth factor binding protein-1 in post-menopausal breast cancer patients. Clin Endocrinol (Oxf). 1995;42(1):23-30.

30. Arcidiacono B, Iiritano S, Nocera A, Possidente K, Nevolo MT, Ventura V, Foti D, et al. Insulin resistance and cancer risk: an overview of the pathogenetic mechanisms. Exp Diabetes Res. 2012;2012:789174.

31. Del Giudice ME, Fantus IG, Ezzat S, McKeown-Eyssen G, Page D, Goodwin PJ. Insulin and related factors in premenopausal breast cancer risk. Breast Cancer Res Treat. 1998;47(2):111-120.

32. Goodwin PJ, Ennis M, Pritchard KI, Trudeau ME, Koo J, Madarnas Y, Hartwick W, et al. Fasting insulin and outcome in early-stage breast cancer: results of a prospective cohort study. J Clin Oncol. 2002;20(1):42-51.

33. Goodwin PJ, Ennis M, Pritchard KI, Trudeau ME, Koo J, Taylor SK, Hood N. Insulin- and obesity-related variables in early-stage breast cancer: correlations and time course 
of prognostic associations. J Clin Oncol. 2012;30(2):164171.

34. Jernstrom H, Barrett-Connor E. Obesity, weight change, fasting insulin, proinsulin, C-peptide, and insulin-like growth factor-1 levels in women with and without breast cancer: the Rancho Bernardo Study. J Womens Health Gend Based Med. 1999;8(10):1265-1272.

35. Kaaks R, Lundin E, Rinaldi S, Manjer J, Biessy C, Soderberg S, Lenner P, et al. Prospective study of IGFI, IGF-binding proteins, and breast cancer risk, in northern and southern Sweden. Cancer Causes Control. 2002;13(4):307-316.

36. Minatoya M, Kutomi G, Asakura S, Otokozawa S, Sugiyama Y, Nagata Y, Mori M, et al. Equol, adiponectin, insulin levels and risk of breast cancer. Asian Pac J Cancer
Prev. 2013;14(4):2191-2199.

37. Pan K, Chlebowski RT, Mortimer JE, Gunter MJ, Rohan T, Vitolins MZ, Adams-Campbell LL, et al. Insulin resistance and breast cancer incidence and mortality in postmenopausal women in the Women's Health Initiative. Cancer. 2020;126(16):3638-3647.

38. Alan O, Akin Telli T, Aktas B, Koca S, Okten IN, Hasanov R, Basoglu $T$, et al. Is insulin resistance a predictor for complete response in breast cancer patients who underwent neoadjuvant treatment? World J Surg Oncol. 2020;18(1):242.

39. Yee LD, Mortimer JE, Natarajan R, Dietze EC, Seewaldt VL. Metabolic health, insulin, and breast cancer: why oncologists should care about insulin. Front Endocrinol (Lausanne). 2020;11:58. 